\title{
Propuesta de caracterización de la renovación urbana en Chile. El caso de la comuna de Santiago Centro
}

Ximena Arizaga. Pontificia Universidad Católica de Chile, Santiago, Chile.

RESUMEN | Se propone una caracterización comprensiva de las políticas de renovación urbana para el caso de Santiago de Chile, teniendo en cuenta para el análisis los textos que están al origen de esas políticas públicas. Se postula la existencia de tres tipos de políticas de renovación urbana que se producen en tres momentos históricos distintos. La propuesta se basa en las diferencias entre esas distintas iniciativas, que tienen en común el propósito de modificar el tejido urbano del centro consolidado de la ciudad mediante la inversión de recursos públicos o el incentivo a la inversión privada. Se analizan tres aspectos para afirmar la existencia de distintas tipologías de renovación: i) el problema que busca resolver el proceso de renovación puesto en vigor; ii) el criterio de selección e intervención en la ciudad escogido por los actores involucrados; iii) y la forma de hacerlo y su expresión física.

PALABRAS CLAVE | renovación urbana, política urbana, historia urbana.

ABSTRACT | A comprehensive characterization of urban renewal policies based on the analysis of the original texts, is proposed for the case of Santiago de Chile. The existences of three types of urban renewal policies that are produced in three different historical moments are found. The proposal is based on the differences between the initiatives that aim to transform the urban fabric of the consolidated city-center by investing public resources or promoting private investment. The analysis focuses on three aspects to affirm the existence of different types of urban renewal in Santiago: i) The problem that seeks to resolve the renewal process; ii) the criteria for selection and intervention in the city, chosen by the actors involved; iii) development and its formal expression.

KEYwORDs | urban renewal, urban policy, urban history.

Recibido el 6 de junio de 2017, aprobado el 21 de noviembre de 2017

E-mail: axarizaga@uc.cl 


\section{Introducción}

En su libro À quoi sert la rénovation urbaine? (2012), Donzelot señala que "lo que produce una política debe leerse primero en lo que la produce, en el proceso de su generación” (p. 3). En esa línea, el objetivo del presente artículo es proponer una caracterización comprensiva de la renovación urbana y su evolución para el caso de Santiago de Chile, basándose en las diferencias que se encuentran al origen de las distintas políticas que tienen por objeto la renovación del centro consolidado. Se postula que comprender las políticas de renovación urbana pasadas permitiría abordar de mejor manera los desafíos de densificación que se presentan hoy en día a la capital chilena, la cual, habiendo urbanizado la totalidad del valle de Santiago, debe repensarse para acoger una población siempre creciente.

Según Lacaze (2010), para entender el urbanismo es pertinente estudiar "las relaciones existentes entre los campos respectivos de la decisión político-administrativa, de la ideología y de las prácticas profesionales" (p. 9). Añade que, en las políticas de renovación urbana, el urbanismo es "una voluntad de acción" con "la perspectiva de ejercer un poder que modifica el espacio de la ciudad" (p. 10). En este acto, la definición del problema, los criterios de decisión y el modo seleccionado para transformar el espacio son esenciales a la constitución de las políticas públicas urbanas y su posterior comprensión.

En este artículo, por "política de renovación urbana” se entenderá una acción del Estado con claras intenciones de modificar el tejido consolidado de la ciudad a través de mecanismos de inversión estatal directa, o del incentivo a la inversión privada. De esta forma, se pretende dejar de lado la natural regeneración de la ciudad, su actualización y embellecimiento. Se analiza el caso de la comuna de Santiago Centro, ya que ofrece un caleidoscopio de ejemplos de renovación urbana que revelan diferentes conceptualizaciones de la intervención del Estado en la ciudad y distintas formas de inversión en el centro consolidado.

Lo que se propone se sitúa en la línea de los trabajos realizados por Le Garrec, Le renouvellement urbain, la genèse d'une notion fourre-tout (2006), Kirszbaum, Rénovation urbaine. Les leçons américaines (2009) y Piron, Renouvellement urbain. Analyse systémique (2002), en el sentido de acotar la existencia de un discurso sobre la renovación urbana en una realidad situada y proponer una caracterización de la misma, que hasta la fecha es inexistente para el caso de Chile, y de Santiago en particular. En consecuencia, la definición de momentos para esta política urbana no se opone a las periodizaciones que han realizado otros estudios sobre la ciudad (Almandoz, 2002; De Ramón, 1990; Gross, De Ramón \& Vial, 1998; Munizaga, 1985; Pavez, 2012; Pérez \& Rosas, 2002; Rosas, Vicuña \& Farías, 2011, entre otros); más bien, responde a la necesidad de establecer tipologías pertinentes para la investigación sobre la renovación urbana.

Se trata, entonces, de estudiar la naturaleza decisional de la política de renovación urbana, lo cual no excluye el mérito de otras investigaciones que pudieran concentrarse en la naturaleza formal de los proyectos que materializan esta política pública. Inspirándose en Lacaze (2010), se analizan tres aspectos para afirmar la existencia de distintas tipologías de renovación urbana para Santiago. Primero, el problema 
que busca resolver el proceso de renovación puesto en vigor; segundo, el criterio de selección e intervención en la ciudad escogido por los actores involucrados en la política de renovación; y tercero, la forma de hacerlo y su expresión física.

El corpus de estudio está constituido por los textos que están al origen de la política de renovación urbana y los proyectos emblemáticos. En este sentido, debe distinguirse la política de renovación urbana del proyecto urbano. La primera busca definir estructuras y principios de organización espacial, mientras que el proyecto urbano corresponde a la propuesta de una realización, basada en planos y cálculos que aseguran su coherencia y materialización (Lacaze, 2010). Por lo tanto, los proyectos no se analizan per se en detalle, sino en sus principios de organización espacial, en tanto reflejo de un orden y materialización de una voluntad de transformar la ciudad.

En el caso chileno, si bien el término "renovación urbana” no siempre fue utilizado, es el finalmente escogido por la legislación nacional (Ley 18595') y en este sentido se conserva como apropiado para referir a las políticas que se analizan a continuación. Al respecto, se postula la existencia de tres tipologías de renovación urbana que se producen en tres momentos históricos distintos:

- Un primer momento, que se extiende desde el plan de transformación de Santiago propuesto por Vicuña Mackenna (1872) al plan diseńado por Brunner (1939), donde prima la necesidad de reestructurar la ciudad existente, ordenarla y embellecerla. El sello del primer plan es la adhesión a principios higienistas, mientras el segundo se rige por una voluntad normativa que marcará el desarrollo de la ciudad durante todo el siglo xx.

- Un segundo momento se manifiesta en las políticas de vivienda de la Corporación de Mejoramiento Urbano (CORMu) y se extiende desde 1965, data de su creación, hasta 1976, fecha de su derogación. Esta política está marcada por el Movimiento Moderno, y se expresa en un conjunto de proyectos de vivienda con una clara visión urbana.

- Un tercer momento corresponde al Plan de Repoblamiento de Santiago, que se inicia con la creación de la Corporación de Desarrollo de Santiago (CORDESAN) en 1985, y se encuentra vigente a la fecha. Esta política busca un equilibrio entre los usos residenciales y el mercado del suelo en la comuna de Santiago Centro, y se implementa a través de un subsidio a la vivienda en zonas definidas de renovación urbana.

Los tres momentos no son continuos. Motivaciones políticas, históricas o de pérdida de interés por el centro de Santiago marcaron tiempos vacíos en la construcción de las políticas de renovación urbana.

La propuesta de tipos de renovación urbana corresponde a una porción de la historia y del proceso político, demográfico y territorial que configura hoy la comuna

1 En 1987 se dicta la Ley 18595 del Ministerio de Hacienda (21/01/1987), que entra en vigencia en 1990 y que faculta al Presidente de la República para declarar Zonas de Renovación Urbana. La Resolución Exenta No 843, del 06/04/1995 del Ministerio de Vivienda y Urbanismo, determina la Zona de Renovación Urbana para la comuna de Santiago y el monto máximo del subsidio directo. 
central de Santiago, y se aproxima a lo que Almandoz (2008, p. 26) define como planning history, en oposición a la urban history centrada en la ciudad y su proceso de urbanización. Esta historia de la planificación, enfocada en los casos de renovación de la comuna central, se inscribe, no obstante, en el proceso de urbanización de Santiago y cobra relevancia en el escenario actual, que invita a repensar formas de densificación y renovación en pos de una mejor calidad de vida para todos los habitantes de la metrópolis. En efecto, si bien Santiago de Chile, con una población total de 6.683 .852 habitantes $^{2}$, es una metrópolis pujante en el contexto latinoamericano (De Mattos, 2004; Fuentes, 2011), también exhibe importantes desequilibrios urbano-territoriales. Sus habitantes, que representan $40 \%$ de la población nacional, se distribuyen de manera dispar en la ciudad, con comunas periféricas que tienen densidades más altas que el centro y pericentro; lo que tensiona las demandas de suelo en estas últimas, mejor equipadas, con mayor acceso a mercados laborales y conectividad que las áreas de expansión.

\section{Tres políticas: diferencias, continuidades y similitudes}

Santiago presenta un rasgo típico de las ciudades donde la renovación urbana es necesaria: su evolución demográfica y urbana combina el fenómeno de expansión de la ciudad con el de contracción de la población en la comuna central. A la vez, presenta un número significativo de casos de renovación urbana que, hasta el momento, no han sido estudiados en su conjunto para definir tipologías que permitan clasificar este tipo de política pública urbana en sus diferencias y similitudes internas, o compararla con la evolución general de las políticas de renovación urbana en el hemisferio norte.

Considerando los criterios de análisis propuestos, se plantea que se pueden diferenciar tres políticas de renovación urbana que, a partir de las acciones a las que dan curso, proponen distintos problemas, criterios muy diferentes de intervención en la ciudad y formas de hacer que se manifiestan en resultados espaciales distintivos hasta el día de hoy. Estos tres tipos de renovación urbana que tienen por territorio la comuna central se resumen en la tabla 1 a continuación, y se explican desde los textos que están al origen de las iniciativas del Estado en el tejido urbano consolidado.

Hasta 1870 , la población chilena vivía mayoritariamente $-80 \%$ aproximadamente- en el campo, pero a fines del siglo XIX y principios del xx se inicia un acelerado proceso de urbanización. Las ciudades crecen y, al mismo tiempo, el auge del salitre (1880-1930) y luego el auge del cobre (1920-1971) permiten al gobierno de Chile una mayor participación en la economía y un aumento en el gasto público, que se traduce en el desarrollo de políticas sociales de mayor alcance en relación con el periodo anterior de construcción de la República (Meller, 1996). 
TABLA I | Resumen de las tipologías de renovación urbana propuestas

\begin{tabular}{|c|c|c|c|c|}
\hline \multirow{2}{*}{ 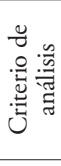 } & \multirow{2}{*}{$\begin{array}{l}\text { Política de } \\
\text { renovación urbana }\end{array}$} & $\begin{array}{l}\text { Los precursores } \\
(1875-1939)\end{array}$ & $\begin{array}{c}\text { La CORMU } \\
(1965-1976)\end{array}$ & $\begin{array}{c}\text { Subsidio de renovación } \\
\text { urbana } \\
(1985 \text {-a la fecha })\end{array}$ \\
\hline & & El damero magnificado & La negación del damero & El damero subvertido \\
\hline \multirow{3}{*}{ 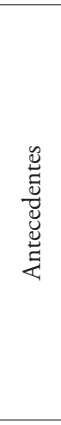 } & \multirow[b]{2}{*}{$\begin{array}{l}\text { Nombre de la políti- } \\
\text { ca pública }\end{array}$} & \begin{tabular}{|l|} 
Plan de Transformación \\
de Santiago (1875) \\
Vicuńa Mackenna \\
\end{tabular} & \multirow[b]{2}{*}{$\begin{array}{l}\text { Corporación de } \\
\text { Mejoramiento Urbano } \\
(1965-1976)\end{array}$} & $\begin{array}{l}\text { Corporación para el } \\
\text { Desarrollo de Santiago } \\
(1985-\text { a la fecha) }\end{array}$ \\
\hline & & $\begin{array}{l}\text { Plan de Transformación } \\
\text { y Plan Regulador } \\
\text { Comunal de Santiago } \\
(1929-1939) \\
\text { Karl Brunner } \\
\end{array}$ & & $\begin{array}{l}\text { Ley } 18595 \text { que declara } \\
\text { Zonas de Renovación } \\
\text { Urbana }(1987 \text { [entra en } \\
\text { vigencia 1990]) }\end{array}$ \\
\hline & Tipo de gobierno & \begin{tabular}{|l|} 
Estado parlamentario \\
$(1891-1925)$ y \\
autoritario \\
$(1927-1931)$ \\
\end{tabular} & $\begin{array}{l}\text { Estado inspirado en el } \\
\text { modelo de bienestar }\end{array}$ & $\begin{array}{l}\text { Estado subsidiario } \\
\text { inspirado en el modelo } \\
\text { de desarrollo neoliberal }\end{array}$ \\
\hline \multirow{2}{*}{ 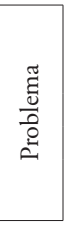 } & Motivaciones & $\begin{array}{l}\text { Sociales (higienistas), } \\
\text { estéticas y funcionales }\end{array}$ & $\begin{array}{l}\text { Sociales y metropolitanas } \\
\text { (equilibrio metropolitano) }\end{array}$ & $\begin{array}{l}\text { Económicas y metro- } \\
\text { politanas (equilibrio } \\
\text { metropolitano) }\end{array}$ \\
\hline & $\begin{array}{l}\text { Problema que busca } \\
\text { resolver }\end{array}$ & $\begin{array}{l}\text { Obsolescencia física y } \\
\text { estética }\end{array}$ & $\begin{array}{l}\text { Déficit de vivienda } \\
\text { Desequilibrios } \\
\text { metropolitanos }\end{array}$ & $\begin{array}{l}\text { Pérdida de población y } \\
\text { de funciones del centro }\end{array}$ \\
\hline \multirow{3}{*}{ 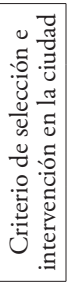 } & \multirow{3}{*}{ Criterio } & \multirow[b]{2}{*}{ Transformación social } & Reestructuración social & $\begin{array}{l}\text { Integración y diversidad } \\
\text { social }\end{array}$ \\
\hline & & & $\begin{array}{l}\text { Integración y diversidad } \\
\text { social (centrado en la } \\
\text { vivienda) }\end{array}$ & Diversidad de usos \\
\hline & & Orden estetizante & $\begin{array}{l}\text { Orden estructurante de } \\
\text { desequilibrios territoriales }\end{array}$ & $\begin{array}{l}\text { Mitigación de } \\
\text { desequilibrios en el } \\
\text { valor del suelo urbano }\end{array}$ \\
\hline \multirow{5}{*}{ 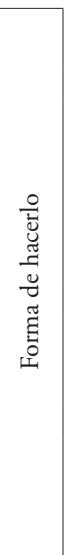 } & Escala & $\begin{array}{l}\text { Escala de ciudad y } \\
\text { escala de barrio }\end{array}$ & $\begin{array}{l}\text { Escala de ciudad y escala } \\
\text { de barrio }\end{array}$ & Escala del lote \\
\hline & $\begin{array}{l}\text { Forma de } \\
\text { intervención }\end{array}$ & Proyecto urbano & Proyecto urbano & $\begin{array}{l}\text { Suma de proyectos } \\
\text { individuales }\end{array}$ \\
\hline & Acción & $\begin{array}{l}\text { Intervención en vías, } \\
\text { espacios públicos y } \\
\text { edificios emblemáticos }\end{array}$ & $\begin{array}{l}\text { Demolición y reconstruc- } \\
\text { ción en grandes conjuntos } \\
\text { habitacionales }\end{array}$ & $\begin{array}{l}\text { Demolición y } \\
\text { reconstrucción lote } \\
\text { a lote }\end{array}$ \\
\hline & Instrumento & $\begin{array}{l}\text { Decanta en un orden y } \\
\text { una norma urbana }\end{array}$ & $\begin{array}{l}\text { Proyectos y planes } \\
\text { seccionales }\end{array}$ & \begin{tabular}{|l|} 
Incentivo financiero \\
(subsidio) acompañado \\
de una norma permisiva \\
\end{tabular} \\
\hline & $\begin{array}{l}\text { Modalidad de } \\
\text { intervención en el } \\
\text { espacio público / } \\
\text { privado }\end{array}$ & $\begin{array}{l}\text { En el espacio público, } \\
\text { para incentivar la } \\
\text { intervención en el } \\
\text { espacio privado } \\
\end{array}$ & $\begin{array}{l}\text { En el espacio privado (la } \\
\text { vivienda) y espacio público } \\
\text { colectivo }\end{array}$ & \begin{tabular}{|l|} 
Operaciones \\
individuales del espacio \\
privado y público \\
adyacente \\
\end{tabular} \\
\hline
\end{tabular}

FUENTE ELABORACIÓN PROPIA 
En este contexto de crecimiento de la población urbana, el final del siglo xix y el principio del xx estuvieron caracterizados por una visión higienista que marcó la política habitacional chilena. En 1906 se dicta la Ley 1838 del Consejo Superior de Habitaciones Obreras, que inauguró la preocupación por la vivienda que cobraría fuerza en décadas posteriores (Hidalgo, 2012). Entonces se produce la primera real transformación de la ciudad como un todo, propuesta por Benjamín Vicuña Mackenna (1831-1886), destacado político e historiador chileno, quien concibe esta transformación a través de una serie de proyectos de infraestructura, espacios públicos y edificios (Pérez \& Rosas, 2002).

Se plantea que este primer tipo de propuesta de intervención sobre el tejido urbano consolidado de Santiago se extiende desde el plan de propuesto por Vicuña Mackenna en La transformación de Santiago (1872), hasta el plan propuesto por Brunner en su Santiago de Chile, su estado actual y futura formación (1930) y el plan regulador de 1939 en el que se plasman sus propuestas.

Si bien el primer plan está marcado por la adhesión a principios higienistas y el segundo se rige por una voluntad normativa que marcará el desarrollo de la ciudad hasta bien entrado el siglo xx, hay continuidad en los principios y en las formas de renovación de la ciudad que postulan. Existe en ambos una preocupación de carácter estético, la cual se fundamenta en prevenciones de salubridad y moralidad que se persiguen en la estructuración de la ciudad, y que buscan transformar Santiago en una urbe moderna. En ambos planes, iniciativas específicas dan cuenta de una visión de la ciudad completa que se materializa en proyectos urbanos de calidad, los cuales, desde el espacio público, promueven la inversión en el espacio privado. Las acciones que proponen se inscriben en un conjunto de proyectos urbanos, y toman como premisa la obsolescencia de lo existente, con una marcada consideración hacia las preexistencias, en particular en las propuestas de Brunner. Si bien se trata-como se muestra en el siguiente apartado- de formas distintas de hacer urbanismo, son similares a la hora de enfrentar la renovación urbana.

Después de este periodo, con la crisis mundial de 1929, se produce un estancamiento de la inversión en la ciudad. Por esos años, las clases acomodadas comenzaron a trasladarse hacia el sector oriente; y paralelamente las clases medias se trasladaron hacia la zona sur, consolidando barrios como Nuñoa y San Miguel. El surgimiento de estas nuevas zonas residenciales se acelera primero con la implementación de los tranvías eléctricos (Castillo, Mardones \& Vila, 2017) y luego con el auge del automóvil (Errázuriz, 2014). Solamente un siglo después del plan de transformación de Vicuña Mackenna se puede distinguir una política de renovación urbana con una lógica distinta.

En 1965, durante el gobierno del Presidente Eduardo Frei Montalva (19641970), se creó el Ministerio de Vivienda y Urbanismo (Ley 16391), y también la Corporación de Mejoramiento Urbano (CORMu), empresa autónoma del Estado cuyas funciones establecidas son, entre otras: "Remodelar o subdividir por cuenta propia o de terceros, terrenos o inmuebles urbanos o rurales para la ejecución de planes habitacionales, equipamiento comunitario y desarrollo urbano" y "Mejorar y renovar las áreas deterioradas de las ciudades, mediante programas de remodelación, rehabilitación, fomento, mantención y desarrollo urbano" (CORMu, 1968, p. 7). Se 
trata esta de la primera expresión de una política con claras intenciones de renovación urbana diferenciada de las políticas de vivienda a cargo de la Corporación de la Vivienda (CORVI, 1953), cuya principal función es "proyectar y construir viviendas económicas y campesinas, por cuenta propia o de terceros" 3 .

Con la impronta formal de la arquitectura moderna, la renovación impulsada por la CORMU apunta a modificar áreas importantes de la ciudad, a través de proyectos completos de barrios que se insertan en el tejido metropolitano consolidado y buscan constituir un contrapeso al proceso de expansión. Estos proyectos, que tienen —al igual que en el caso anterior - una visión completa de la ciudad, se inscriben en un proceso de estructuración urbana que es también preocupación social; pero esta vez desde la vivienda, espacio privado, hacia el espacio público. Su implementación se basa en la tabula rasa, con cierta continuidad en la consideración de las permanencias urbanas, que se expresa en el detalle de los proyectos urbanos. Su escala de intervención es el barrio, pensado como una unidad, generando espacios colectivos de recreación y circulación que soportan las torres de vivienda.

Con posterioridad al golpe de Estado de 1973, se impone la idea de que el Estado debe reducirse, adoptando un mero rol subsidiario. Los principios globales que lo rigen son la vigencia del mercado, apertura al comercio exterior y un modelo descentralizado, donde los agentes toman decisiones independientemente de la autoridad central. Los incentivos económicos se consideran esenciales para aumentar la productividad, la inversión y el ahorro; el mercado es el mecanismo óptimo de asignación de recursos y el Estado solo debe intervenir en corregir o eliminar distorsiones, limitándose el gasto público. En 1976, la cormu y la CORVI son remplazadas por el Servicio de Vivienda y Urbanización (sERviu), orientado solamente a la provisión de vivienda. Se inicia una fase en la que el Estado se autoexcluye en materia de producción del espacio urbano, dejando paso al modelo de desarrollo neoliberal. Se retorna a la zonificación por usos de suelo y a la normativa como principales instrumentos, y se abandona el proyecto urbano como forma de intervención. En este modelo, el Estado deja a merced del mercado la producción de la ciudad, interviniendo solamente con proyectos puntuales ${ }^{4}$ que, si bien participan en la actualización y embellecimiento del espacio público, no pueden considerarse como una política de renovación urbana.

Se había producido, entonces, un fenómeno contradictorio de desarrollo acompańado con deterioro en la comuna central: por un lado, la línea 1 del metro motivaba la renovación física de las edificaciones; por otro, la congestión, la degradación ambiental y la obsolescencia física de las construcciones producían la migración de las familias, acentuando la percepción de abandono, en particular en horarios no laborales (Valenzuela, 1994). En la década de 1980, el Municipio de Santiago toma consciencia de la situación de depresión del centro. Esta podía agravar no solo los problemas sociales, sino también la economía local, dado que las principales

3 D.s. 508 del minvu, 26/10/1966, fija las funciones especiales de la CORvi.

4 El alcalde de Santiago (designado por el Régimen Militar), Patricio Mekis (1976-1979), remodeló y peatonalizó el paseo Ahumada, la calle de la Bolsa de Comercio y la calle Nueva York, las calles Estado, Tenderini y Phillips y la plazoleta frente al Teatro Municipal, transformó el Palacio Cousiño en museo, y reconstruyó el museo de la Casa Colorada, entre otras obras. 
actividades financieras se concentraban todavía en la comuna central, lo que afectaba el precio del suelo y la salud financiera del mismo municipio. Los estudios de entonces arrojaron que los patrones de localización estaban mutando, las sedes comerciales y financieras habían comenzado a migrar hacia otras áreas de la ciudad (El Golf y posteriormente Nueva Las Condes); la industria había migrado hacia la periferia y el comercio comenzó a ubicarse entonces en los nuevos corredores de transporte (Valenzuela, 1994). Ante la preocupación unánime por la situación de deterioro que sufría la comuna, la que se acentuó con el terremoto de 1985, se creó la Corporación para el Desarrollo de Santiago (CORDESAN), una entidad privada con personalidad jurídica, sin fines de lucro, cuyo objetivo es mejorar la calidad de vida de vecinos y usuarios de la comuna. La CORDESAn promueve el desarrollo social y urbano de la comuna de Santiago, fomentando la colaboración entre la I. Municipalidad de Santiago, el sector privado y la comunidad.

En 1987 se dictó una ley que entró en vigencia en 1990, la que faculta al Presidente de la República para declarar Zonas de Renovación Urbana ${ }^{5}$. El Ministerio de Vivienda y Urbanismo ${ }^{6}$ determina la Zona de Renovación Urbana para la comuna de Santiago y el monto máximo de subsidio directo. En 1991, la Municipalidad de Santiago publicó Propuesta de desarrollo para la renovación de Santiago, con el lema "Santiago es tarea de todos". La propuesta plantea tres temas relevantes, con sus respectivas líneas de acción: i) la necesidad de ampliar el rol residencial de la comuna; ii) el fomento de actividades de servicio, comercio e industria; y iii) el mejoramiento de la calidad de vida de la población. Finalmente, en esta política, que es explícitamente de renovación urbana, destacan las que son hoy las grandes temáticas de este mecanismo de intervención en la ciudad: la diversidad social y la diversidad de usos.

Con base en una problematización que toma origen en el deterioro, el despoblamiento y la ineficiencia en la asignación de equipamientos y costos urbanos a nivel metropolitano, el criterio de intervención es el incentivo financiero para propiciar la acción de privados. En este caso, la política se materializa lote a lote, lo que deja de lado el proyecto urbano y la visión de ciudad que estaba contenida en las otras dos tipologías de renovación urbana. El subsidio se apoya en una norma que liberaliza las alturas de edificación, fomentando la inversión en el espacio privado y dejando la intervención en el espacio público a cargo del promotor inmobiliario.

\section{Tres momentos de la renovación urbana}

\section{Los precursores: el damero magnificado}

El primer momento corresponde al proceso de emergencia del urbanismo como disciplina reflexiva, crítica y científica, "distinta de las artes urbanas anteriores" (Choay, 2004 , p. 8). En Chile, el urbanismo no emerge como crítica al proceso de industrialización, que a fines del siglo XIX es todavía muy incipiente, sino ya apropiado como disciplina ordenadora de la ciudad y de la sociedad. El nuevo orden busca adaptar

5 Ley 18595 del Ministerio de Hacienda (21/01/1987).

6 Resolución Exenta No 843, del 06/04/1995, luego modificada el 21/06/1999. 
la sociedad a la estructura y no viceversa ${ }^{7}$, y recoge todos los principios que Choay señala en su periodización: racionalización de las vías de comunicación, creación de estaciones de ferrocarril y tranvías, especialización de los distintos sectores de la ciudad. La ciudad se comienza a pensar por zonas: barrios industriales, barrios residenciales, barrios obreros, y se inicia su progresiva suburbanización (Choay, 2004).

En el caso de Santiago, este proceso se realiza en dos tiempos, que ejemplifican también la diferencia que hace Choay entre el preurbanismo y el urbanismo. El primer tiempo corresponde al plan para la transformación de Santiago, de Vicuña Mackenna, que se define en 1872 pero cristaliza en 1910, cuando se realizan las principales obras junto con la celebración del primer centenario de la Nación. El segundo tiempo está marcado por la propuesta de "futura formación" de Santiago, de K. Brunner (1932), y su definición de los principios básicos del primer Plan Regulador Comunal de Santiago de 1939, que se mantendrá vigente -aunque con múltiples modificaciones- hasta 1985.

La propuesta urbana de Vicuña Mackenna es una intervención política, con una visión explícita de la sociedad que se quiere alcanzar; en la propuesta de Brunner, en tanto, la intervención urbana se despolitiza y se explicita en un discurso científico. Este último, propiamente disciplinar, primará posteriormente en el quehacer del Estado sobre la ciudad, centrándose en la norma como expresión de la práctica del urbanismo.

En el primer discurso sobre la renovación de la capital, el de Vicuña Mackenna, la ciudad es considerada, por primera vez desde su fundación, como un conjunto. En un contexto de crecimiento de la población urbana por las migraciones desde el campo y los campamentos mineros, el plan está pensado "no solo para embellecer a Santiago sino para salvarlo" (p. 7); vale decir, sus intenciones no son meramente formales, sino también estructurantes: "para que la capital de la república sea digna de su nombre i de su misión” (p. 10) ${ }^{8}$.

Dos obras caracterizan especialmente el plan de Vicuña Mackenna: la canalización del río Mapocho y el camino de cintura. En estas dos medidas destacan el deseo de orden, la infraestructura como principio rector de la modernidad, las vías como base de racionalización de la ciudad y la plusvalía del suelo como un objetivo explícito.

La construcción de los Tajamares del Mapocho, que se terminan en 1802, resolvió el problema de las inundaciones y permitió, a fines del siglo XIX, repensar una amplia zona de la ciudad (Castillo, 2014). La canalización del río, señala Vicuña Mackenna en La transformación de Santiago, tiene entre sus objetivos: " 1. ” Incorporar a la ciudad propia, formando un solo cuerpo con ella, los hermosos i hoi relegados barrios de ultra Mapocho. 2. ${ }^{\circ}$ Prolongar las calles laterales de la ciudad, uniéndolas con las de aquellos barrios por puentes de poca estensión (64 metros de largo) i mediano costo” (pp. 12-13). Además de sus propósitos particulares, estas iniciativas permitieron la venta de nuevos terrenos -los que hoy en día bordean el Parque Forestal- que, al valorizarse, aseguraron el financiamiento de las obras.

7 Como es el caso en la transformación de París conducida por Haussmann, donde se busca adaptar la ciudad a la nueva sociedad burguesa que la habita (Choay, 2004).

8 En las citas de Vicuña Mackenna se ha respetado la grafía del original. 
El segundo proyecto más importante de la propuesta de Vicuña Mackenna es el camino de cintura, cuya "realización daría a nuestra capital el más hermoso boulevard del mundo" (p. 161). Para el autor, esta vía define "la ciudad propia” y la distingue de los arrabales. Su diseño es estudiado en detalle, tramo por tramo, por distintas comisiones de ingenieros: se establece el ancho, el espacio de calzada y aceras (8 a 10 metros), la arborización (cuatro filas de árboles), el potencial de completarla por líneas férreas; vale decir, se definen no solo las condiciones formales del proyecto, sino también su función en el transporte de la ciudad y su futuro crecimiento. Este gesto estructura y define la comuna central hasta nuestros días, su vialidad y zonificación: establece los límites de los servicios urbanos; y un "cordón sanitario", por medio de la vegetación, crea una serie de paseos en el contorno y define zonas para las fábricas. A través de estas dos acciones, el plan modifica el tránsito y ordena la estructura de Santiago centro, lo cual, se suma al proyecto de alcantarillado que se ejecuta en 1910 y "modifica en completitud la trama central" (Rosas, 2006, p. 47).

Este proyecto de camino de cintura, que define "la ciudad ilustrada, opulenta, cristiana” (Vicuña Mackenna, 1872, p. 24), ponía en valor otro espacio de la ciudad: la Alameda. En este sentido, es destacable una visión de conjunto que interrelaciona obras para conseguir una visión total de ciudad. El plan de transformación es un proyecto cuyo modelo es París, lo que se explicita claramente en el deseo de Vicuña Mackenna de convertir el eje principal de la Alameda de las Delicias en los "Campos Eliseos de esta capital" (p. 136). Ilustra el anhelo modernizador y civilizador que caracteriza la época: "[que] las cuatro avenidas hoi solo diseñadas en el papel, surcadas por la locomotora, se conviertan en verdaderos muros de la civilización" (p. 136). Se trata, sin duda, de un plan sin precedente, cuya completitud hace sombra a planes y propuestas posteriores.

Además de estas obras mayores, el plan consideraba un programa muy completo de obras de mejoramiento de barrios desde una perspectiva no solo estética y formal, sino como respuesta a "una de las más vitales necesidades de la ciudad" (Vicuña Mackenna, 1872, p. 24). La propuesta apuntaba al orden, pero también a resolver problemas de salud de la población, y contiene en su esencia un deseo moralizador que no será completamente abandonado en el proyecto de Brunner. Propone así la "Destrucción COMPleta de todo lo Que existe" i la promulgación de leyes i ordenanzas que obliguen a los que especulan” (p. 28). La política erige una norma finalmente, lo cual no es muy distinto de lo que propondrá Brunner medio siglo más tarde.

A través de estos gestos mayores y puntuales, Santiago pasa de ser una ciudad colonial a ser una ciudad moderna. El plan se expresa en el plano de Ansart de 1875 (figura 1b); sus cambios son visibles en el plano posterior de Tornero (figura 1a) y marcan un contraste notorio con el plano anterior de Herbage (figura 1c). 
FIgURA I | Estructuración de Santiago en el plan de transformación de Vicuña Mackenna

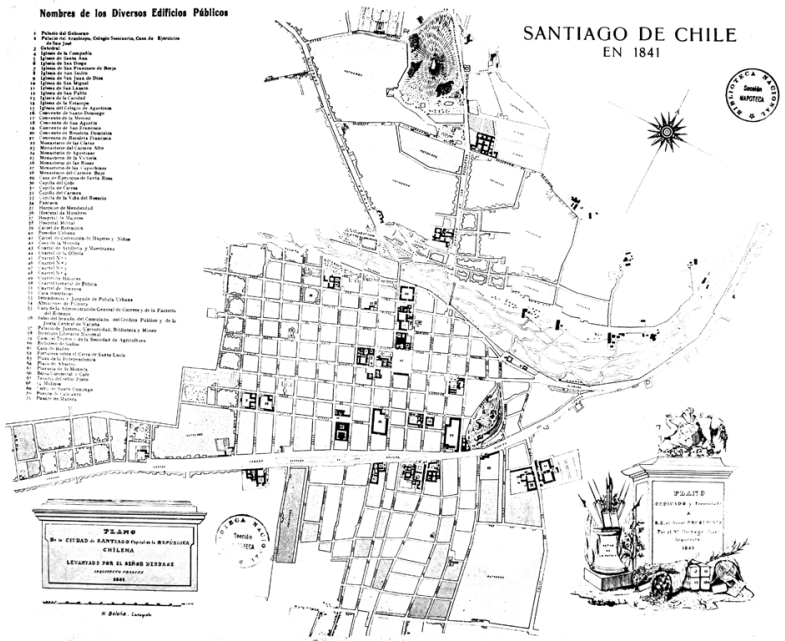

(1a) Plano de Herbage (1841)

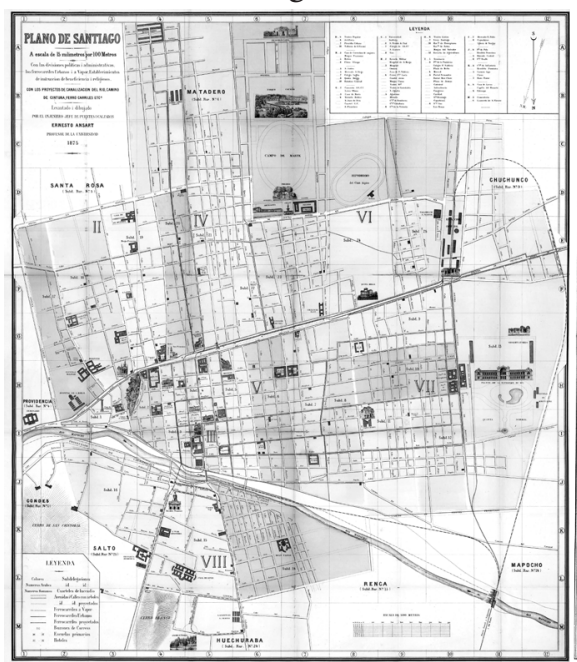

(1b) Plano de Ansart (1875)

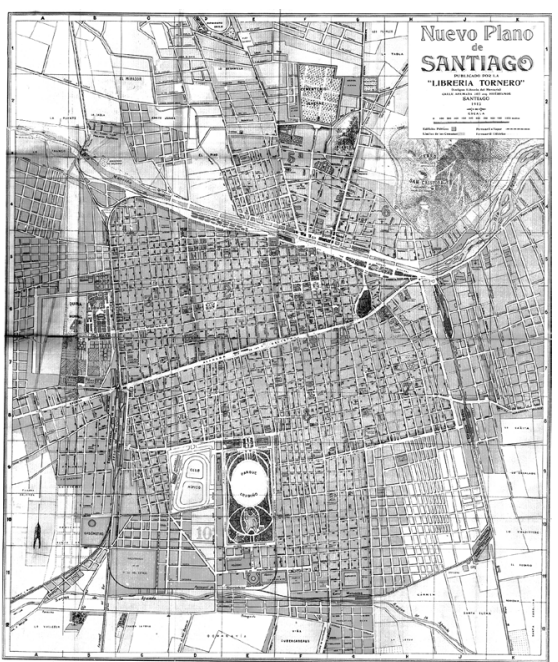

(1c) Plano de Tornero (1912)

FUENTE COLECCIONES BIBLIOTECA NACIONAL DE CHILE, EN HTTP://WWW.ARCHIVOVISUAL.CL

Aunque el resultado arquitectónico y morfológico fue distinto, el plan propuesto para la transformación de Santiago por K. Brunner (1932) cristaliza y ordena principios que fueron dispuestos por el intendente de Santiago en el siglo xix. Lo que Brunner propone es "una reorganización local sin cambios sustanciales en la estructura urbana ya existente" (Hofer, 2006), transformando un "hacer" en "norma”. A esto se suma un cambio disciplinar: la aparición del urbanismo como ciencia, y la emergencia de la zonificación por usos de suelo como su principio rector. 
Karl Brunner (1887-1960), arquitecto-urbanista vienés, fue asesor del Gobierno de Chile entre 1929 y 1933, bajo la presidencia de Carlos Ibáñez del Campo (19271931) y Arturo Alessandri Palma (1932-1938). En 1934 fue contratado por la Municipalidad de Santiago, para elaborar un anteproyecto de plan regulador. Su asesoría contribuyó a la definición de una normativa de construcciones y urbanización que se plasmó finalmente en la Nueva Ley General sobre Construcciones y Urbanización, que surgió con posterioridad al terremoto de Talca de 1928.

El periodo del plan propuesto por Brunner está marcado por un gobierno autoritario ${ }^{10}$, que realizó importantes inversiones en obras públicas para paliar la crisis de $1929^{11}$. La construcción se vio entonces favorecida por una norma que, en 1933, suprimió temporalmente los impuestos al sector, convirtiéndose este rubro en el más próspero de la época. La ciudad de Santiago creció por el auge de la industria manufacturera y la migración desde las minas salitreras.

En este segundo periodo disciplinar, el interés de los urbanistas, antes historiadores, políticos y economistas, se desplaza de la preocupación social hacia estructuras técnicas y estetizantes (Choay, 2004). Entre los posibles modelos de planificación, en ese entonces Chile no escogió el camino radical liderado por Le Corbusier, sino un modelo respetuoso de las permanencias urbanas. En el proceso, el urbanismo se fue asimilando cada vez más a la norma y a su expresión más radical: la monozonificación por usos; así, en el camino fue perdiendo otros aspectos de sus virtudes constituyentes de política pública.

Bajo principios todavía estetizantes, la propuesta de Brunner no representa un quiebre con el periodo anterior; se trata de un proceso de ordenación y embellecimiento de la ciudad que se propone en forma normada y que busca principalmente solucionar, a través de una política pública, problemas del espacio construido (figura 2c). Las propuestas de Brunner apuntan a un mejoramiento en el estándar del espacio público, sus usos comerciales y recreativos: no solamente es diseñado hasta en su detalle de mobiliario, sino que también se proponen nuevas vías (figura $2 \mathrm{~b}$ ) y perforaciones al interior de las manzanas (figura 2c). Vialidad, ensanche y zonificación son los principios rectores de esta transformación, sin que esto signifique un abandono de la preocupación por la forma y composición. La vivienda aparece como la base de todo estudio urbanístico (Brunner, 1939, Tomo I, p. 71); y se verá el carácter vinculante que cobraría en los períodos posteriores de renovación urbana (figura 2a). Su discurso, en este ámbito, es muy similar al de Vicuña Mackenna: la vivienda insalubre es deficiente por sus aspectos higiénicos y también morales, que afectan no solo a quien la habita, sino a la sociedad en su conjunto (pp. 233-234).

10 El gobierno de Ibáñez (1927-1931) "de autoritarismo incuestionable”, "había puesto en marcha un programa de obras públicas a gran escala. Entre 1928 y 1931, La Moneda invirtió casi 760 millones de pesos en diversos proyectos: desagües, caminos, puentes, barracas, prisiones, pistas de aterrizaje, instalaciones portuarias, 500 kilómetros de ramales para ferrocarriles, la nueva fachada sur de La Moneda, la residencia de verano del presidente en Viña del Mar” (Collier \& Satter, 1998, p. 195).

11 La gran depresión de 1929 afectó fuertemente al país: Chile fue descrito como el país del mundo más devastado por la crisis. La caída de los precios del salitre y del cobre significó una importante migración desde el norte a las ciudades de la zona centro (Collier \& Satter, 1998). 
En Santiago de Chile, su estado actual y futura formación (1932), Brunner destaca con fuerza la norma y el orden como fin. El objetivo del plan para Santiago de Chile, es "la necesidad urgente del saneamiento y mejora de los barrios hoy depreciados, y ante todo la preparación técnica de todo un plan del desarrollo de la ciudad cuya base deberá ser la distribución orgánica de las zonas según su distinta destinación" (p. 15). Esta combinación entre la norma y la zonificación tendría a largo plazo consecuencias nefastas — entonces impredecibles - que se formalizan en la siguiente política de renovación urbana.

En la propuesta de K. Brunner, la modernidad es también el principio rector. En este proyecto de ciudad están muy presentes y entremezcladas dos escalas: i) la escala territorial, las vías en tanto conectoras, los parques; y ii) la escala del proyecto urbano, los edificios y su construcción, el tratamiento delicado de las manzanas (figura 2c), las vías en tanto espacio público de la ciudad (figura 2b). El proyecto urbano y el urbanismo están todavía muy entreverados: el proceder no es puramente de política pública, sino fondo y forma, al igual que en Vicuna Mackenna.

\section{FIgURA 2 Transformación de Santiago en el plan de Brunner}

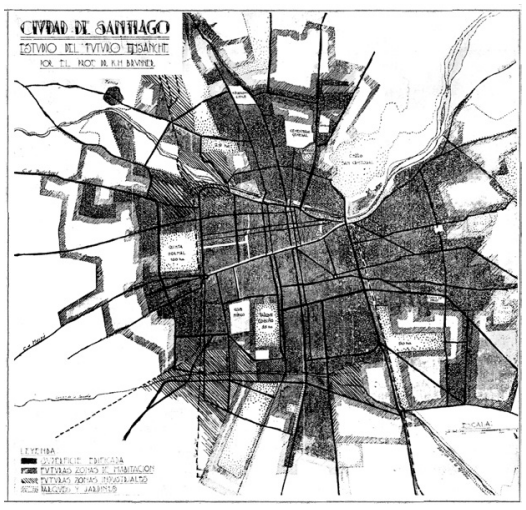

(2a) Plan Brunner (1939)

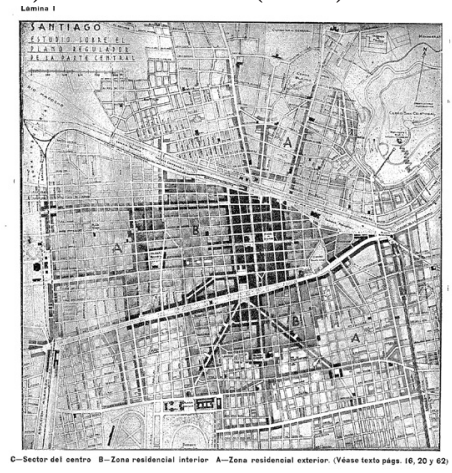

(2b) Propuesta para el Barrio Cívico

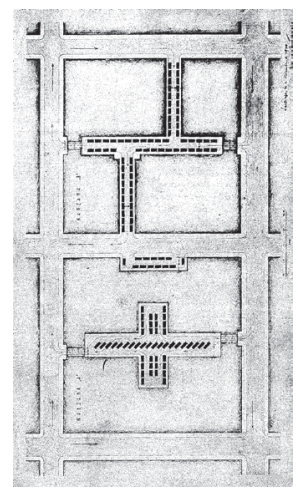

(2c) Formación de estacionamientos al interior de la manzana

FUENTES (2A) MINISTERIO DE LA VIVIENDA Y URBANISMO, I 996;.(2B) MINISTERIO DE LA VIVIENDA Y URBANISMO, I996; (2C) BRUNNER, I939, TOMO II 
CORMu, el déficit de vivienda y la negación del damero

El proceso de renovación urbana impulsado por la CORMU se enmarca en un modelo que toma origen en el Congreso Internacional de Arquitectura Moderna (CIAM), y que se caracteriza por un desplazamiento del urbanismo hacia la arquitectura y la aplicación de esta disciplina a la escala urbana (Luque, 2004). Con la impronta formal de la arquitectura moderna, la renovación urbana impulsada por la CORMU propone modificar áreas importantes de la ciudad a través de proyectos rupturistas e integrales que, analizados en detalle, muestran una voluntad de dialogar e incorporarse a la ciudad preexistente.

Esta política de renovación urbana se produce en el seno de un Estado inspirado en el modelo de Bienestar que se venía gestando desde fines del siglo xix con el auge del salitre (1880-1930) y posteriormente con el auge del cobre (1920-1971). Se trata no solamente de otro momento económico, político y disciplinar, sino también de otro momento social. Para ese entonces, Santiago ya no era una ciudad incipiente, con una sociedad dominada únicamente por la élite; se trataba, por el contrario, de una ciudad en plena expansión, donde se expresaba la sociedad de masas (Romero, 2010 [1976]).

En esa época, los habitantes acomodados ya habían comenzado a trasladarse hacia la cordillera, lo que tuvo como consecuencia que el centro de Santiago se desplazara hacia la zona oriente, y la comuna fuera perdiendo su carácter residencial. En ese contexto, con la creación de la CORMu se inició una nueva lógica de renovación urbana, donde se manifiesta la voluntad de aportar cambios sustanciales tanto a la ciudad como a la sociedad. En esta política comparecen, por un lado, la preocupación estructural, territorial e institucional por una ciudad en la que se produce una "incontrolada extensión horizontal" (MINVu, 1969, p. 2), que sobrecarga las infraestructuras y servicios; y por otro, la preocupación por otorgar a la población planes habitacionales que incluyan accesibilidad a equipamientos urbanos en un entorno de calidad (figura 3).

La situación ya observada por Brunner (1930), de despoblamiento combinado con un alto precio del suelo, se acentúa nuevamente en este periodo. La cormu hace manifiesta esta preocupación en sus objetivos, que apuntan en particular a constituir un contrapeso al proceso de expansión: "La tarea, que significa reconstruir los sectores deteriorados y mal aprovechados de la ciudad, aumentando su densidad habitacional, liberando una alta proporción del suelo respectivo para fines de higiene ambiental y de recreación de sus habitantes, y aprovechando su ubicación central para el uso más eficiente del equipamiento urbano y de la infraestructura actuales, representa una conquista que enorgullece a Chile" (CORMu, 1969, p. 15).

Opera entonces la modalidad de proyecto urbano, al igual que en el período anterior, y se configura una visión de conjunto sobre la ciudad que se transparenta tanto en los planos como en el discurso (figura 3). Las intervenciones se realizan sobre grandes paños de terreno, configurando barrios que se plasman en los planes seccionales y buscan un "diálogo entre el habitante y las posibilidades que ofrece la ciudad” (Auca 16, 1969a, p. 75). En esos espacios la torre no es el objeto neutro y aislado promovido por el movimiento moderno; bien por el contrario, es un objeto que dialoga con Santiago y su geografía (figura 3). 
FIGURA 3 | Remodelación San Borja
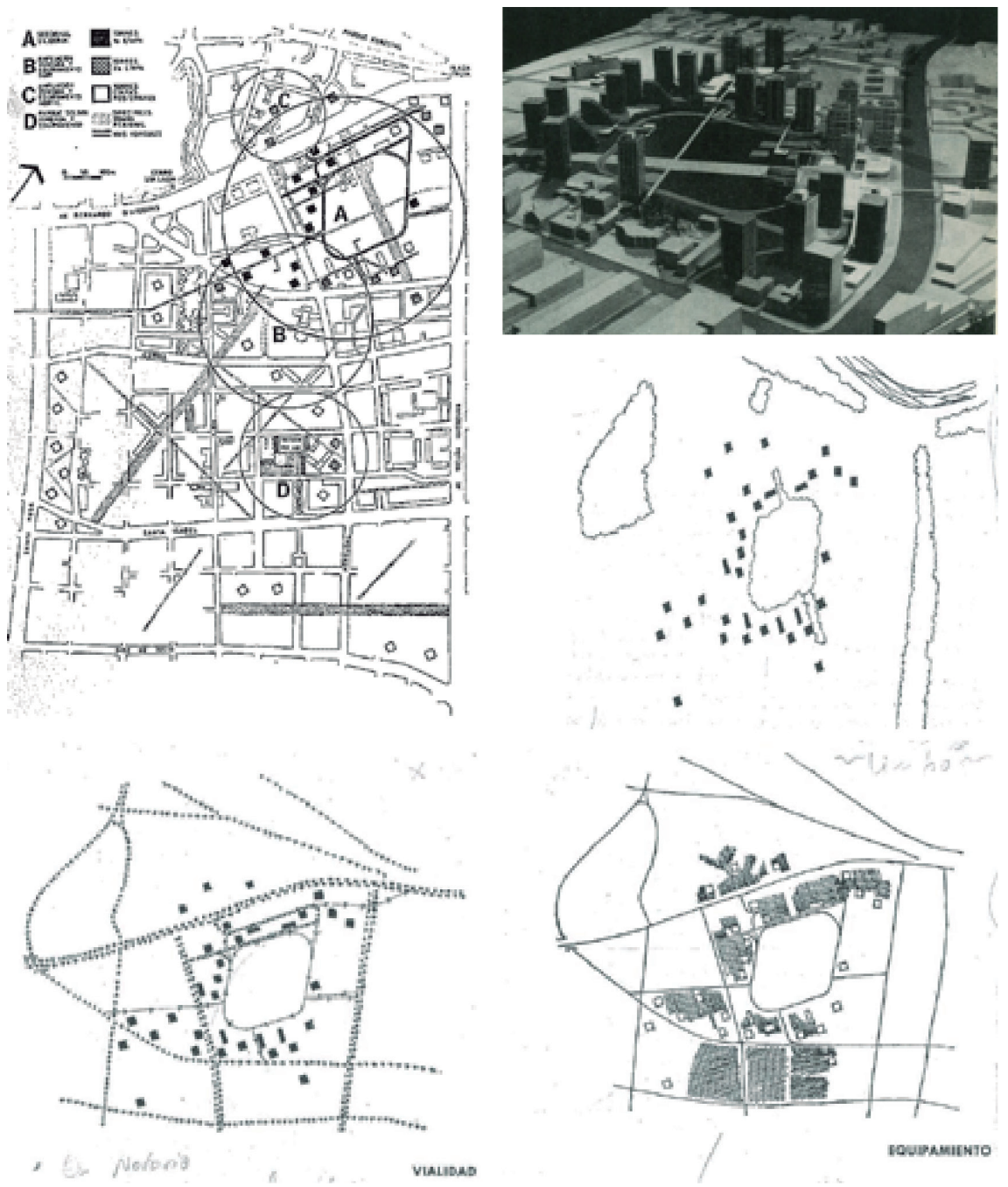

FUENTE AUCA (I6), I969B

(...) Una solución óptima que consiste básicamente en un anillo que toma contacto a través del equipamiento con los puntos urbanos de mayor intensidad y le da continuidad a las unidades habitacionales, liberando un espacio interior que cohesiona y le da sentido comunitario a las viviendas propuestas, obteniendo así un área que es claramente urbana sin perder su calidad vecina. (...) El anillo de contacto propone una vialidad peatonal liberada del tráfico vehicular, con diferentes niveles que participan tanto de la ciudad misma como del área verde interior (Auca $\left.\mathrm{N}^{\circ} 16,1969 \mathrm{~b}\right)$. 
Subsidio de renovación urbana: la ciudad "sorpresa": el damero subvertido

La política de repoblamiento que caracteriza el tercer momento de la renovación urbana se basa en lo que fue el espíritu de las Políticas Nacionales de Desarrollo Urbano (PNDU) de 1979 y 1985. La primera, de 1979 (véase MINVu, 2012), establece la orientación del instrumento de planificación hacia el mercado. Señala que se entenderá por "desarrollo urbano" el "proceso de concentración espacial de las actividades económicas y sociales de la población, a partir de densidades que permiten captar las economías externas de la aglomeración” (p. 14). En la PNDU de 1979 se hacen "explícitas" las siguientes declaraciones: "El suelo no es un recurso escaso" y "queda definido por su mayor rentabilidad" (p. 16), lo que conlleva la declaración de que "debe regirse por disposiciones flexibles, definidas por los requerimientos del mercado” (p. 17). La renovación urbana, desde esta perspectiva, se entiende debe ser promovida por el Estado, y realizada por privados: "para lo cual el Gobierno proveerá las normas técnicas y legales y los estímulos tributarios necesarios que la hagan factible" (p. 15).

En Santiago, dos documentos propiciaron la creación del Subsidio de Renovación Urbana que aplica para 38 áreas centrales de distintas ciudades del país. El primero, Imperativo de la recuperación urbana: algunos costos de la expansión (CORDESAN, 1987), hace hincapié en el problema de ineficiencia económica al que ha conducido la expansión urbana "al no lograr una asignación óptima de los recursos” (p. 5). Señala que, al contrario, las ventajas de renovación del centro son el uso intensivo de la infraestructura existente, la posibilidad de revertir el deterioro del área, la promoción de nuevas actividades mixtas y la atracción de población, con beneficios directos para las familias (empleo, escolarización). Con base en esta propuesta se estudian incentivos económicos que favorezcan la recuperación señalada. El segundo documento, Costo nacional diferencial de localizar viviendas básicas (Curso Interamericano en Preparación y Evaluación de Proyectos de Inversión [Ciaper], 1985), analiza la renovación potencial de Santiago Poniente y estudia los sectores que serán beneficiados por un incentivo económico.

Un tercer estudio, elaborado por la Pontificia Universidad Católica de Chile, Estudio para el Plan de Desarrollo Urbano y Económico de Santiago (1988), define las áreas prioritarias de intervención, a partir de un análisis muy detallado. Señala que se trata de renovar "sectores intersticiales que aparecen indiferenciados, morfológicamente no definidos y ambiguos, sin elementos de configuración determinantes", que son "condicionantes de una imagen deteriorada para toda la comuna" y se estima "poseen un gran potencial, en términos de definir elementos de configuración, conectándose con áreas o fragmentos homogéneos, o de constitución de sus bordes" (p. 32). Existe en esta conceptualización un fuerte condicionamiento de la renovación por la obsolescencia física del conjunto edificado como principio de producción, lo que condujo a la destrucción del patrimonio modesto de la ciudad (figura 3a). En 1991, la I. Municipalidad de Santiago publicó Propuesta de desarrollo para la renovación de Santiago, con el lema "Santiago es tarea de todos". De acuerdo con este documento, el primer eje de acción relativo a ampliar el rol residencial de la comuna apunta a "la heterogeneidad social, a través de una política de promoción inmobiliaria [que] impulsará la renovación y remodelación, la reutilización de 
viviendas y de sitios eriazos y la densificación de áreas de baja densidad poblacional"; y a la heterogeneidad de usos "con el fin de consolidar la vida de los barrios" (p. 27).

En 1990 se aprobó el nuevo Plan Regulador Comunal (PRC) de Santiago, que deroga parte de los artículos del Plan Brunner de 1939. La modificación de la norma permitió un drástico cambio en la relación entre la altura de edificación y el espacio público. En este proceso, se pierde el cuidado por las permanencias y se subvierte la escala de la cuadrícula original en su tercera dimensión: la altura. El espacio público es abandonado a su suerte, tanto en la norma como en los proyectos banalizados de los desarrolladores inmobiliarios. El resultado es una ciudad "sorpresa"12, en la que edificaciones de alta densidad se insertan en una trama urbana que no estaba prevista para resistir tales presiones (figura 4). Habiendo abandonado completamente el proyecto urbano, aparecen aglomeraciones de edificios que no buscan dialogar con la ciudad preexistente, y menos hacerse cargo de los impactos que provocan (López-Morales, Arriagada-Luco, Gasic-Klett \& Meza-Corvalán, 2015).

En esta lógica de renovación urbana, el espacio público es resuelto por cada promotor inmobiliario como espacio residual. Los edificios contienen en su interior espacios comunes: piscinas, quinchos, salones y gimnasios.

La renovación urbana aparece entonces muy marcada por el planning; domina la relación entre el levantamiento de datos, su análisis y la propuesta de un plan de acción, y se abandona la escala del proyecto urbano. El instrumento es una ley orientada exclusivamente a la promoción de la renovación urbana mediante la definición de zonas en las que se aplica un subsidio. Se omite el proyecto de ciudad: se privilegian los objetivos y el procedimiento por sobre el modo como se alcanzan, desatendiendo el objeto al que se aplica el plan: el espacio urbano.

FIGURA 4 | Transformación de Santiago y subsidio de Renovación Urbana

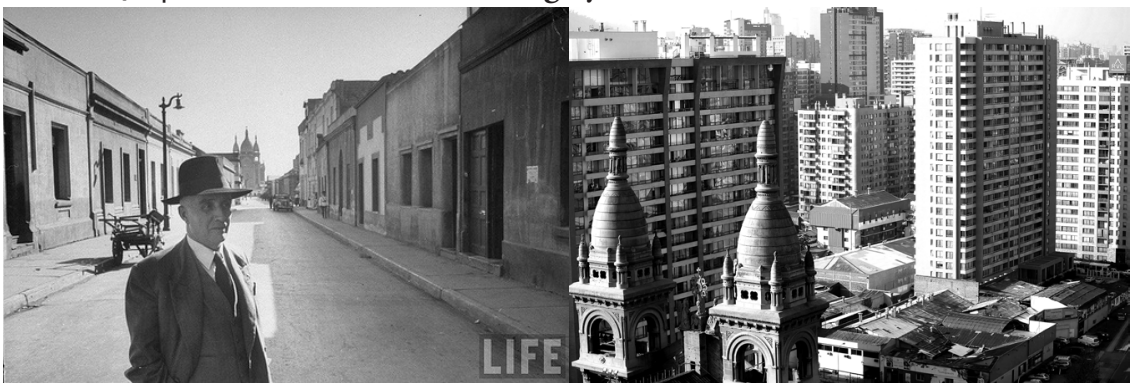

(4a) Barrio Santa Isabel, 1950

(4b) Barrio Santa Isabel, 2012

FUENTES (4A) REVISTA LIFE (HTTP://WWW.FLICKRIVER.COM/PHOTOS/28047774@NO4/7788738750/); (4B) EL DÍNAMO (HTTPS://WWW.ELDINAMO.CL/NEGOCIOS/2OI $7 / 07 / \mathrm{I} 7 /$ POR-QUE-LASPERSONAS-VALORAN-LOS-DEPARTAMENTOS-DE-3O-METROS-CUADRADOS/)

12 Seminario: "Interrogantes frente al crecimiento de la ciudad de Santiago", realizado por la Comisión de Urbanismo de la Cámara Chilena de la Construcción en junio de 1990, poco después de entrar en vigencia el subsidio de Renovación Urbana. Alberto Collados Baines, entonces presidente de la Comisión, señala: "La comuna de Santiago ha logrado, por fin, aprobar un nuevo Plan Regulador. (...) se llegó a la más positiva de las opciones, a una ciudad cuya fisonomía será el producto sorpresa de los edificios que se vayan creando, frente a una ciudad con una fisonomía preconcebida que luego se va rellenando" (Collados, 1990, p. 11). 


\section{Consideraciones finales}

La propuesta presentada permitió discernir el surgimiento del concepto de renovación urbana y su evolución para el caso de Santiago de Chile entre las principales políticas sobre la ciudad, y el discurso de los principales actores que intervienen. Este ejercicio reviste interés, dado que permite observar y distinguir las lógicas que operan en el discurso urbano local en relación con la evolución de este fenómeno a nivel global; y, a la vez, acotar el concepto de renovación urbana en oposición a otros procesos de actualización de la ciudad. Tal perspectiva obligó a interesarse en las continuidades entre las políticas de renovación urbana y en los quiebres entre unas y otras, factores que llevaron a que la investigación abordara un arco temporal extenso, en el sentido de longue durée propuesto por Fernand Braudel (1958).

En el caso de Santiago, se hace evidente el condicionamiento de la ciudad al valle que terminó por ocupar íntegramente (Pérez, 1995); y es también la finitud del valle la que condiciona y obliga a pensar la ciudad desde una perspectiva de nueva inversión en el centro y en el pericentro consolidados. La relación con este paisaje, a la vez extenso y finito, estará presente en todas las políticas urbanas, explícita o implícitamente. Los proyectos, en particular desde la segunda mitad del siglo $\mathrm{xx}$, tomarán el paisaje como testigo del crecimiento y desarrollo de Santiago, con la cordillera al fondo como garante y anhelo de una vida sana, explícitamente en el caso de los proyectos cormu e indirectamente en los proyectos de torres que prometen esta vista lejana y extensa.

Las intervenciones topográficas del valle -la canalización del Mapocho, la supresión del sistema de captación de aguas al oriente del cerro Santa Lucía (las Cajitas de Agua), la fractura de la ciudad que introdujo la autopista Norte-Sur y la contención al interior del cinturón Américo Vespucio- condicionaron las políticas urbanas y modificaron la situación inicial del espacio geográfico. La ciudad actual ya no se ubica en un valle agrícola extenso; por el contrario, la mancha urbana se extiende hasta los faldeos cordilleranos y ha agotado su suelo agrícola. Una situación de tensión se produce entre un intramuros -al interior del cinturón Américo Vespucio- dotado de servicios y equipamientos, y una periferia menos aventajada, como en la mayoría de las grandes ciudades del mundo. Esta situación es la que justifica reflexionar sobre las políticas de renovación urbana ya implementadas, y de las cuales es posible observar las consecuencias que han tenido sobre la ciudad, para pensar la renovación urbana futura.

La primera política de renovación urbana se caracteriza, en ambos planes -el de Vicuña Mackenna y el de Brunner- por el deseo de estructuración urbana y social. La renovación urbana se centra en el espacio público o en la construcción de edificios destacados, que luego impulsarían la inversión privada. Si bien en los dos se reconoce la importancia de la vivienda, no se centran en la solución de su déficit, sino en una solución unificada para la ciudad de Santiago. Difieren, entonces, de las dos políticas de renovación urbana siguientes, en las cuales la provisión de viviendas estará al centro del problema que busca resolver la intervención.

La zonificación por usos de suelo en forma latente, y luego en forma explícita en el Plan Regulador propuesto por Brunner, será el eje rector de la planificación, 
tendiendo a su radicalización y al monouso, que luego se convertiría en uno de los peores síntomas del decaimiento de Santiago centro, junto con la aparición de la ciudad segregada.

La segunda política de renovación urbana, al igual que la anterior, trata no solamente de la ciudad, sino que es también una propuesta de renovación de la sociedad. Se diferencia de la primera porque centra su principio rector en el déficit de vivienda, motivada por la resolución de desequilibrios urbanos de gran escala. En esta política opera la modalidad de proyecto urbano y la introducción de una nueva escala de edificación y cantidad de unidades de vivienda, que transformó el espacio público en espacio de circulación, con múltiples propósitos y posibilidades indefinidas. Aunque marcada por el planning y el funcionalismo, la aplicación local de estas corrientes internacionales será matizada en los proyectos más emblemáticos de la CORMu. Estos proyectos de corte "moderno" no asumirán plena indiferencia hacia la ciudad existente, respetando las permanencias, la geografía y el contexto urbano, con cierta continuidad de los aprendizajes dejados por Brunner.

En estas dos primeras políticas de renovación urbana, el Estado buscará conjugar las acciones de dominio público con las ventajas del mundo privado en la ejecución. La obsolescencia se incorpora como causa y motivo permanente de la renovación: se justifica a través de la higiene en Vicuña Mackenna; se basa en la pérdida de vigencia estética en Brunner; y en el periodo de la CORMu, en los cambios de funcionalidad. Ambos tipos de renovación propusieron la demolición de amplios sectores de la ciudad; no obstante, los precursores no rompieron con el manzanero tradicional y sublimaron el espacio público, mientras que, en el periodo de la cormu, se superpuso la intervención negando la manzana tradicional.

La tercera política de renovación urbana analizada, si bien no adopta la tabula rasa como solución formal, termina alcanzando una negación mayor del damero y el espacio público. A pesar de que los estudios previos que se realizan para definir el instrumento de esta política son sensibles a las voces del posmodernismo, finalmente, al estar fuertemente marcados por la obsolescencia como principio de producción y la tendencia a asimilar el patrimonio a la monumentalidad, terminan proponiendo la desaparición del patrimonio modesto de la ciudad. La suma de acciones individuales no concertadas, a la escala del lote y su espacio adyacente inmediato, tiene un impacto todavía por dimensionar.

Esta política, se postula, es sin embargo heredera de la primera: priman el zoning y la norma como principios rectores. En este caso, la norma se aplica sin proyecto urbano, a la neutralidad del damero. En el camino se pierde el cuidado por las permanencias y se subvierte la escala de la cuadrícula original en su tercera dimensión. El espacio público, que había sido confinado a su rol principal de circulación en el proyecto moderno, es ahora abandonado a la solución propuesta por el desarrollador del lote. En la norma y en los proyectos ejecutados, los espacios propuestos al peatón resultan ser espacios residuales y banalizados, mientras que para el uso comunitario se entregan espacios de uso privativo.

En los tres casos está presente la renta del suelo, abordada de distintos modos. En el caso de los dos primeros planes de transformación, se incentiva la inversión privada y, por lo tanto, la valorización del suelo, a través de la intervención en el 
espacio público. En el caso de la política promovida por la CORMU, la construcción de proyectos de vivienda en el centro de la ciudad busca equilibrar el acceso a suelo de precio elevado para familias de estratos medios, mediante la participación del Estado en la gestión y desarrollo del suelo urbano. Por último, el subsidio de renovación urbana persigue en su esencia compensar el precio del suelo mediante este aporte a la compra de viviendas, incentivando de esta forma la inversión privada y el repoblamiento del centro ${ }^{13}$.

Los espacios resultantes de estas tres políticas de renovación urbana siguen vigentes en la ciudad, son utilizados cotidianamente, se actualizan y entremezclan entre sí. En el caso de los barrios resultantes del primer momento de renovación de Santiago, como Lastarria-Bellas Artes, por ejemplo, su evolución positiva da cuenta del grado de consolidación y flexibilidad que introdujeron los planes de transformación de Vicuña Mackenna y Brunner. Los sectores objeto de las intervenciones de la CORMU conocieron momentos de deterioro, que se acrecentaron por las dimensiones de la unidad de intervención; y hoy en día, son objeto de un interés creciente por parte de nuevos residentes motivados por su espacialidad y ubicación central (Arizaga, 2016). Por último, los barrios donde se concentra el subsidio de renovación urbana ofrecen un panorama incierto en sus posibilidades de evolución e integración de las escasas preexistencias restantes (Arizaga, 2017), lo que preocupa a los habitantes y los contrapone a la sobreposición armónica de construcciones que se produjo en los dos casos anteriores.

La necesidad de renovación del pericentro de Santiago debería buscar soluciones mixtas, que tomen de cada una de las políticas de renovación anteriores las soluciones que han demostrado ser mejores en el tiempo. Estudiar los procesos de renovación urbana, sus principios rectores y formas de hacer, permite, por lo tanto, comprender el valor presente y las consecuencias de más de un siglo de políticas de renovación urbana.

\section{Agradecimientos}

Este artículo ha sido realizado en el marco de un post-doctorado financiado por el Centro de Desarrollo Urbano Sustentable (cedeus) en la línea "Entorno Construido".

13 Cabe señalar que el último censo arrojó resultados muy positivos en el crecimiento de la comuna central, la que pasó de tener 208.197 habitantes en el censo 2002 (311.415 en el precenso 2012), a 404.495 habitantes en el censo 2017. 


\section{Referencias bibliográficas}

Almandoz, A. (ed.). (2002). Planning Latin America's capital cities, 1850-1950. Londres: Routledge.

Almandoz, A. (2008). Entre libros de historia urbana: para una historiografia de la ciudad y el urbanismo en América Latina. Caracas: Equinoccio.

Arizaga, X. (2016, agosto). El concepto de ambiente: su uso para la comprensión del espacio público. El caso de Santiago Centro: Más de un siglo de políticas de renovación urbana. Tesis para optar al grado de Doctor en Arquitectura y Estudios Urbanos, Pontificia Universidad Católica de Chile. En http://repositorio.conicyt.cl/handle/10533/209639

Arizaga, X (2017). La renovación urbana y el patrimonio de la ciudad: tres barrios de Santiago. La Voz de La Chimba (41), 35-36. En http://www.ciudadviva.cl/wp-content/ uploads/2017/12/18.-XArizaga.pdf

Auca, $\mathrm{N}^{\circ}$ 16. (1969a). ¿Edificación en altura? Transcripción del debate realizado por Auca el 14 de mayo de 1969. Auca (16), 60-70.

Auca, № 16. (1969b). Remodelación San Borja. Auca (16), 71-88.

Braudel, F. (1958). Histoire et Sciences sociales: La longue durée. Annales. Économies, Sociétés, Civilisations, (13-4), 725-753. En http://www.persee.fr/doc/ahess_0395-2649_1958_ num_13_4_2781

Brunner, K. (1930). Conceptos urbanísticos de Santiago. Conferencia inaugural del Seminario de Urbanismo, dada en el Salón de Honor de la Universidad de Chile, el 6 de mayo de 1930. Anales de la Universidad de Chile, 8(abr.-jun., serie 2), 872-906. https://doi. org/10.5354/0717-8883.2012.25569

Brunner, K. (1932). Santiago de Chile, Su estado actual y su futura formación. Santiago de Chile: Imprenta La Tracción.

Brunner, K. (1939). Manual de Urbanismo. 2 Tomos. Bogotá: Ediciones del Concejo de Bogotá.

Castillo, S., Mardones, M. \& Vila, W. (2017). El Estado sobre ruedas. Transporte público, politica y ciudad. La ETCE 1945-1981. Santiago: Ediciones Universidad Alberto Hurtado.

Choay, F. (2004, [1965]). L’Urbanisme. Utopies et réalités. París: Éditions du Seuil.

Collados, A. (1990). Intervención de don Alberto Collados Baines, Presidente de la Comisión de Urbanismo, en el Seminario Interrogantes frente al crecimiento de la ciudad de Santiago. En Boletín Estadístico [Cámara Chilena de la Construcción, CCHC], 17(210), 1.3-1.9.

Collier, S. \& Satter, W. (1998 [1996]). Historia de Chile 1808-1994. Madrid: Cambridge University Press.

Corporación para el Desarrollo de Santiago (CORDESAN). (1987). Imperativo de la recuperación urbana: Algunos costos de la expansión. Documento de trabajo realizado por A. Ureta, L. Musalem, J. Domeyko, M. E. Hofer \& N. Sostin. Santiago, Chile: cordesan.

Curso Interamericano en Preparación y Evaluación de Proyectos de Inversión (CIAPEP). (1986). Costo nacional diferencial de localizar viviendas básicas. Santiago, Chile: Instituto de Economía, Pontificia Universidad Católica de Chile.

De Mattos, C. (2004). Santiago de Chile: Metamorfosis bajo un nuevo impulso de modernización capitalista. En C. de Mattos, M. E. Ducci, A. Rodríguez \& G. Yáñez (eds.), Santiago en la globalización: ¿una nueva ciudad? (pp. 17-46). Santiago, Chile: Ediciones SUR / EURE libros. En http://www.sitiosur.cl/r.php?id=16 
De Ramón, A. (1990). Santiago de Chile 1941-1991. Historia de una sociedad urbana. Santiago, Chile: Sudamericana.

Donzelot, J. (2012). À quoi sert la rénovation urbaine? París: Presses Universitaires de France (PUF).

Errázuriz, T. (2014). La administración de Ibáńez del Campo y el impulso a la circulación moderna (Santiago, 1927-1931). Historia, 47(2), 313-354. https://doi.org/10.4067/ S0717-71942014000200002

Fuentes, L. (2011). Ciudad competitiva ¿̇ciudad cohesionada? Vinculaciones entre competitividad urbana y cohesión social en Santiago de Chile. En C. de Mattos, W. Ludeńa \& L. Fuentes, Lima-Santiago. Reestructuración y cambio metropolitano (pp. 209-236). Santiago, Chile: Instituto de Estudios Urbanos y Territoriales, Pontificia Universidad Católica de Chile / Lima, Perú: Centro de Investigación de la Arquitectura y la Ciudad, Pontificia Universidad Católica del Perú.

Gross, P., De Ramón, A. \& Vial, E. (1984). La imagen ambiental de Santiago 1880-1930. Santiago, Chile: Ediciones Universidad Católica de Chile.

Hofer, A. (2003). Karl Brunner y el urbanismo europeo en América Latina. Bogotá: Áncora.

Ilustre Municipalidad de Santiago (IMs). (1991). Propuesta de desarrollo para la renovación de Santiago. Santiago, Chile: Ediciones Ims.

Kirszbaum, T. (2008). Rénovation urbaine; les leçons américaines. París: Presses Universitaires de France (pup).

Lacaze, J. P. (2010 [1990]). Les méthodes de l'urbanisme. París: Presses Universitaires de France (PUF).

Le Garrec, S. (2006). Le renouvellement urbain. La genèse d'une notion fourre tout. La Défense, France: Éditions du PUCA (Plan Urbanisme Construction Architecture).

López-Morales, E., Arriagada-Luco, C., Gasic-Klett, I. \& Meza-Corvalán, D. (2015). Efectos de la renovación urbana sobre la calidad de vida y perspectivas de relocalización residencial de habitantes centrales y pericentrales del Área Metropolitana del Gran Santiago. EURE, 41(124), 45-67. https://doi.org/10.4067/S0250-71612015000400003

Luque, J. (coord.). (2004). Constructores de la ciudad contemporánea. Aproximación disciplinar a través de los textos. Navarra: Departamento de Urbanismo de la Escuela Técnica Superior de Arquitectura, Universidad de Navarra / Editorial Dossat.

Martínez, R. (2007). Santiago de Chile: los planos de su historia: siglos xvi a xx: de aldea a metrópolis. Santiago, Chile: Ediciones Facultad de Arquitectura, Urbanismo y Paisaje, Universidad Central de Chile.

Meller, P. (1996). Un siglo de Economía Política chilena (1890-1990). Santiago, Chile: Andrés Bello.

Ministerio de Vivienda y Urbanismo (MINvu) (1968). La Corporación de Mejoramiento Urbano. Santiago, Chile: Ministerio de Vivienda y Urbanismo, Edición cormu.

Ministerio de la Vivienda y Urbanismo / Colegio de Arquitectos de Chile (1996). Juan Parrochia Beguin Premio 1996: 6 planes para Santiago. Santiago, Chile: Arquitaller.

Ministerio de Vivienda y Urbanismo (mINvu). (2012). Hacia una Nueva Politica Urbana para Chile. Vo1. 1 | Antecedentes históricos. Santiago, Chile: minvu / Políticas Nacionales de Desarrollo Urbano. En http://cndu.gob.cl/wp-content/uploads/2014/10/ Antecedentes-Hist\%C3\%B3ricos1.pdf 
Munizaga, G. (1985). Estructura y ciudad. Santiago, Chile: Ediciones Universidad Católica de Chile, Vicerrectoría Académica.

Muñoz Malushka, L. (1937). La transformación de las ciudades chilenas. Revista Zig-Zag (Santiago, Chile), Edición extraordinaria: "Arquitectura, Construcción, Urbanismo", 96-97.

Pérez, F. \& Rosas, J. (2002). Cities within the city: Urban architectural transfers in Santiago de Chile, 1840-1940. En A. Almandoz (ed.), Planning Latin America's capital cities, 18501950 (pp. 109-138). Londres: Routledge.

Pérez, F. (1995). Santiago entre su origen y sus desafios. En P. Bannen Lanata (ed.), Santiago de Chile: quince escritos y cien imágenes (pp. 19-26). Santiago, Chile: ARQ.

Pontificia Universidad Católica de Chile (PUC). (1988). Estudios para el plan de desarrollo urbano y económico de la comuna de Santiago. Coordinado por Gustavo Munizaga. Santiago, Chile: Facultad de Arquitectura y Bellas Artes, Facultad de Ciencias Económicas y Administrativas, Ilustre Municipalidad de Santiago, Secretaría de Planificación y Coordinación.

Romero, J. L. (2010 [1976]). Latinoamérica. Las ciudades y las ideas. Buenos Aires: Siglo Veintiuno.

Rosas, J. (2006). Conformación y consolidación del centro de Santiago 1930-1960. En M. Saavedra \& M. Laborde (eds.), Santiago centro: Un siglo de transformaciones (pp. 4053). Santiago, Chile: Ediciones Ilustre Municipalidad de Santiago, Dirección de Obras Municipales.

Valenzuela, J. (1994). Urban decay and local management strategies for the metropolitan centre: the experience of the Municipality of Santiago, Chile. En C. P. Hoshino, K. Oya, A. L. Fernández \& M. Cassim (eds.), Latin American regional development in an era of transition: the challenges of decentralization, privatization and globalization (pp. 65-85). United Nations Centre for Regional Development (UNCRD) Research Report Series No. 8. Nagoya, Japan: United Nations Centre for Regional Development.

Vicuña Mackenna, B. (1872). La transformación de Santiago: Notas e indicaciones respetuosamente sometidas a la Ilustre Municipalidad, al Supremo Gobierno y al Congreso Nacional. Santiago, Chile: Imprenta de la Librería del Mercurio, de Orestes L. Tornero. En http://www.memoriachilena.cl/602/w3-article-86624.html 